\title{
Es cuestión de criterio
}

\section{Reseña de: Frederic, Sabina (coordinadora). De la desmilitarización a la profesionalización. Un estudio etnográfico sobre la formación básica de la Policía Federal Argentina. Bernal: UNQ -Publicaciones Ciencias Sociales, 2016.}

\author{
Inés Oleastro \\ Centro Interdisciplinario de Metodologia de las Ciencias Sociales - \\ Instituto de Investigaciones en Humanidades y Ciencias Sociales (CIMeCS-IdIHCS)- \\ Universidad Nacional de La Plata. \\ Universidad Nacional de Quilmes-CONICET, Argentina ineoleastro@gmail.com
}

¿Cuál es la relación entre la formación policial y el ejercicio de la profesión? ¿Cuál es el perfil policial que se espera de esa formación? ¿Cuáles son los "valores policiales"? ¿Son distintos en un momento que en otro? ¿Qué pasa con la labor policial en la comisaría? Son los sentidos comunes alrededor de la Policía Federal Argentina (PFA) los que motorizan este trabajo: enfrentarlos y discutirlos es el desafío que aquí se proponen los/as siete antropólogos/as que lo impulsan: Frederic, Galvani M., Bover, Calandrón, Galvani I., Melotto y Ugolini. Nos encontramos entonces con el presupuesto sobre la identidad policial, aquella que pareciera forjarse durante la formación y por lo tanto que es allí donde hay que poner el foco de las reformas para transformar sus prácticas. Con esta visión conviven así concepciones sobre la autonomía de la policía y la idea de la corrupción policial, que se intenta explicar a través de la militarización de esta fuerza. Todas estas preguntas y afirmaciones se suspenden para poner el terreno policial en cuestionamiento, desarmarlo y leerlo desde una perspectiva etnográfica, en sus propios términos y sus propios tiempos.

La investigación que supone este libro surge a partir de un convenio entre el Ministerio de Seguridad de la Nación y la Universidad Nacional de Quilmes. El primero, por entonces a cargo de Nilda Garré, buscaba conocer en profundidad el estado de situación en materia de formación y capacitación policial para llevar adelante diferentes reformas y políticas alrededor de estos puntos. A partir de ello, y en consonancia con otros trabajos previos en relación a antropólogos/as que se impulsaron desde su gestión, se buscaba un abordaje más integral que comprendiera la perspectiva de los actores y la cotidianeidad policial. El trabajo fue entonces el primer estudio etnográfico sobre la Escuela se Cadetes, la Escuela de Agentes, las comisarías de CABA y cuerpos de la PFA. El mismo implicó un trabajo colectivo que potenció el acercamiento a los distintos actores, donde las diferencias de género, de edad y de jerarquía académica fueron la llave para construir confianza y referencia con los distintos sujetos a trabajar. De esto se desprende, además, un proceso constante de articulación desde el diseño del proyecto hasta la versión final de los informes, desafío no menor y poco abordado en el hacer etnográfico.

Hay entonces en este trabajo dos puntos interesantes de debate, que pertenecen a distintas dimensiones y claves analíticas, pero que resultan fundamentales para hacer posible esta investigación. Por un lado, el lugar de la antropología y el trabajo etnográfico en la escena de las políticas públicas; ¿qué punto de vista, qué trabajo específico y qué enfoque puede aportar un/a antropólogo/a en un problema de este tipo? ¿Cómo se pone en juego el carácter académico y político de una misma investigación? ¿Cuál es la distancia y relación entre el conocimiento generado por un equipo de trabajo de ciencias sociales y la política que del mismo deriva? Y el segundo eje de análisis, que responde en cierto punto las preguntas anteriores, tiene que ver con el cuestionamiento de los sentidos comunes, no solo imperantes en la sociedad en su conjunto sino también 
en las autoridades ministeriales y los funcionarios en general alrededor de la formación policial y el ejercicio de la profesión.

Respecto al primer punto y en la especificidad de este aporte que la antropología tiene para dar, que en este caso se da en el encuentro específico entre el Ministerio y la Universidad, el trabajo abre el debate sobre la intervención y el compromiso de la disciplina, para pensar y reforzar la importancia del rol etnográfico en el diseño de políticas públicas. De aquí se desprende el posicionamiento de los/as investigadores/as respecto a las instituciones que convocan y respecto al campo mismo. Por otro lado la confianza sobre esa producción de conocimiento que se le concede a un/a antropólogo/a en este tipo de intervenciones, que en este caso conlleva a la formación de un grupo de trabajo. Y por último la pertenencia a otras dependencias institucionales, en este caso la UNQ, es lo que permite y potencia un acercamiento corriéndose de la imagen de funcionarios público. Esto último es fundamental para diferenciar entre quienes buscan conocer y quienes buscan generar medidas a partir de ello, elemento que además influye en el vínculo a construir y la confianza en el trabajo de campo en este caso con los policías.

Intervenir desde la antropología implica pararse en un punto intermedio entonces entre aquello que el Ministerio pide, busca y motoriza al convocarlos/as, y entre aquellas preguntas académicas que se traen desde las universidades. Entre estos dos puntos, no por ello opuestos, los/as cientistas sociales se mueven en diálogo constante. Es allí donde surgen nuevas preguntas, nuevos problemas, que permiten reorientar y responder a todas las partes, que veremos que, en este caso, lleva a descubrimientos que se corren de los presupuestos manejados por quienes convocaban al trabajo. Este tipo de investigaciones traen consigo una serie de requerimientos, de procedimiento, de confidencialidad y de identidad para con los actores en el campo también.

En este sentido, este primer punto da lugar a aquel segundo eje que mencionábamos: el presupuesto de la relación entre la formación policial y el ejercicio posterior de la profesión, que parte del mismo Ministerio y que se cuestiona en esta investigación.

A lo largo del trabajo se describen todas las dimensiones que intervienen en la formación de cadetes y de suboficiales, y luego también en su práctica profesional. En este sentido, no sólo se abordan las prácticas mismas, en las clases, los espacios comunes y las comisarías, sino también los sentidos que los actores describen de las mismas así como de la institución y sus normas ¿Cuáles son los saberes adquiridos? ¿Qué aptitudes se pretende entrenar? ¿Cuáles son las prácticas pedagógicas y la distribución curricular? Son sólo algunas de las preguntas en torno a la vida en las Escuelas. Todas estas se enmarcan en la forma en que desde la institución se proyectan y tramitan las reglas, en la administración de los espacios y los tiempos en la escuela y los vínculos con los/as otros/as/.

Lo que se busca de los alumnos durante la Escuela de Oficiales es el desarrollo de determinadas destrezas y saberes que deben ser adquiridos durante los tres años de formación policial. En ese proceso se construye y se espera un determinado perfil: los alumnos son sometidos a pruebas y cursos orientados a aplicar la profesión policial en sus términos ideales, todo lo que para ellos/as debe ser un policía. Aquí no sólo entra entonces una formación amplia en el área disciplinar del derecho sino también del uso y control de la fuerza, la formación en tiro y el estado físico.

En sus propios discursos y sus propios términos, ese ideal es el que debe ser alcanzado y su vínculo con la vida profesional la principal herramienta en la formación policial. Para esto último entonces, aparecen las pasantías en comisarías como el puente fundamental de contacto entre los aprendizajes en la Escuela y la práctica de las comisarías. Es esta una de las instancias más valoradas por alumnos e instructores, a pesar de sus falencias.

Es interesante ver que, además, la cuestión de la conducción y del mando aparece como aptitud a incorporar para el trabajo policial. Es así que, a través de distintas normativas y prácticas, los cadetes aprenden a ser conducidos y a conducir desde que ingresan a la institución. La jerarquía y la autoridad resultan entonces centrales en la construcción de la subjetividad profesional para quienes luego quieran insertarse en las 
comisarías. A partir de sistemas de mando, de responsabilidades por año de cursada y el orden de méritos se van introduciendo estas nociones que, en sus propios discursos, parecen fundamentales.

A través de distintas nociones como la colonización del tiempo, el espiritu de cuerpo y el criterio se pone en el centro el ser/hacer policial para los propios actores. A partir entonces de una exhaustiva descripción de la Escuela, de los procesos de aprendizaje y del trabajo en la comisaría, el trabajo nos invita a cuestionar este vínculo que parecía tan directo entre la formación y el ejercicio policial. Es entonces la Escuela un espacio idealizado y separado, y la comisaría un lugar que se presenta como distante de aquella. En los primeros años de labor un policía se encuentra preparado para sus tareas, en tanto ocupa el lugar de Oficial de Guardia, para lo que es formado y preparado en los tres años previos. Sin embargo, la práctica policial que implica toda la carrera, los distintos roles en la comisaría a lo largo del tiempo, no son aprehendidos durante el pasaje por la Escuela, sino más bien en la calle, pisando el terreno en la propia práctica. Es aquí que los propios policías localizan que se forja el criterio, noción que deriva en un saber práctico, un saber hacer policial que le permite desenvolverse en su trabajo.

Finalmente, en el encuentro entre estos dos ejes que se destacan de la singularidad del trabajo aparece el aporte más que significativo que este grupo de cientistas sociales hace al problema. A partir del balance y el análisis que surge del trabajo de campo etnográfico, llegan las propuestas para el Ministerio, poniendo foco en las cuestiones a mejorar, a fortalecer y a revisar. Hay entonces dos principios fundamentales sobre los que se trabaja; el primero tiene que ver con la jerarquización de la formación básica para mejorar la calidad educativa; y el segundo con identificar cuáles son las continuidades y rupturas entre esta formación y el ejercicio policial.

Resulta pertinente partir de estos ejes por un análisis sobre la intervención del Estado argentino en términos de inversión y tiempo, así como de quienes estudian para convertirse en policías; y en relación con las intenciones y los objetivos que el Ministerio presentaba para llevar adelante esta investigación. Es así que, a través de este estudio etnográfico se logran visualizar los problemas nodales de la formación y de su relación del desempeño profesional policial, y ponerlos así en diálogo con respuestas y modificaciones acordes a los perfiles que el propio Ministerio buscaba incorporar para estos profesionales.

De aquí surge entonces no sólo una descripción detallada y exhaustiva del cotidiano policial, de sus puntos de vista y de las heterogéneas concepciones sobre su formación y ejercicio profesional, sino también propuestas concretas para el abordaje que posteriormente sería implementado por el Ministerio de Seguridad, en un diálogo con sus propios actores. Esto no sólo encuentra su potencial a partir del reconocimiento de los problemas significativos, sino que permite que estas modificaciones se construyan en diálogo y sean viables para ambas partes. 